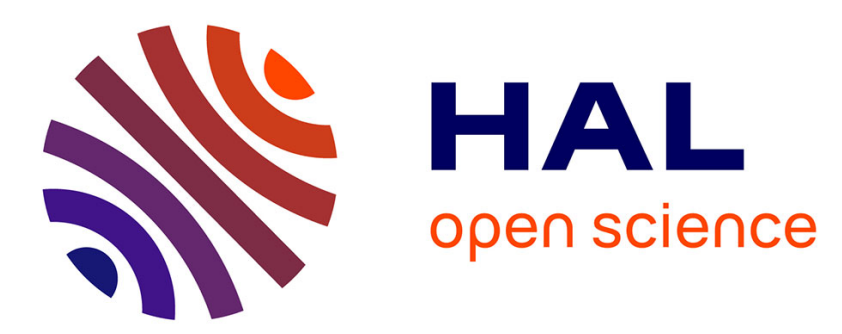

\title{
Observation of negative line tensions from Plateau border regions in dry foam films
}

\author{
J.-C. Géminard, A. Żywocinski, F. Caillier, P. Oswald
}

\section{To cite this version:}

J.-C. Géminard, A. Żywocinski, F. Caillier, P. Oswald. Observation of negative line tensions from Plateau border regions in dry foam films. Philosophical Magazine Letters, 2004, 84 (3), 10.1080/0950083010001646707 . hal-01314366

\section{HAL Id: hal-01314366 https://hal.science/hal-01314366}

Submitted on 11 May 2016

HAL is a multi-disciplinary open access archive for the deposit and dissemination of scientific research documents, whether they are published or not. The documents may come from teaching and research institutions in France or abroad, or from public or private research centers.
L'archive ouverte pluridisciplinaire HAL, est destinée au dépôt et à la diffusion de documents scientifiques de niveau recherche, publiés ou non, émanant des établissements d'enseignement et de recherche français ou étrangers, des laboratoires publics ou privés. 


\title{
Observation of negative line tensions from Plateau borders regions in dry foam films.
}

\author{
J.-C. Géminard, A. Żywociński , F. Caillier, and P. Oswald. \\ Laboratoire de Physique de l'E.N.S. de Lyon, 46 Allée d'Italie, 69364 Lyon Cedex. \\ - Institute of Physical Chemistry, Polish Academy of Sciences, PL-01-224 Warsaw 42, Poland.
}

\begin{abstract}
We measure the angles produced in the Plateau border region of "dry" soap films. In a simple experimental geometry, we demonstrate that a negative line tension can be attributed to these regions. This result has important consequences for the theoretical description of foams approaching the dry limit.
\end{abstract}

\section{INTRODUCTION.}

Foams are collections of gas bubbles trapped in a complex network of thin liquid or solid films and they have a great deal of practical importance in industrial processes as well as in the everyday life (food, shaving foam, insulators... ) [1]. Establishing the relations between the physical properties of the films and gas, and those of the resulting material remains a fascinating, yet puzzling, problem which has motivated numerous experimental and theoretical studies. Interestingly, understanding foam physics exhibits the same types of challenges as those one is faced with when studying granular materials. In these materials, the relations between the individual properties of the solid grains and their collective behavior are still only partially understood [2]. In foams, the problem is further complicated by the possibility for the gas bubbles to deform, which necessitates complete understanding of the local geometry in these systems.

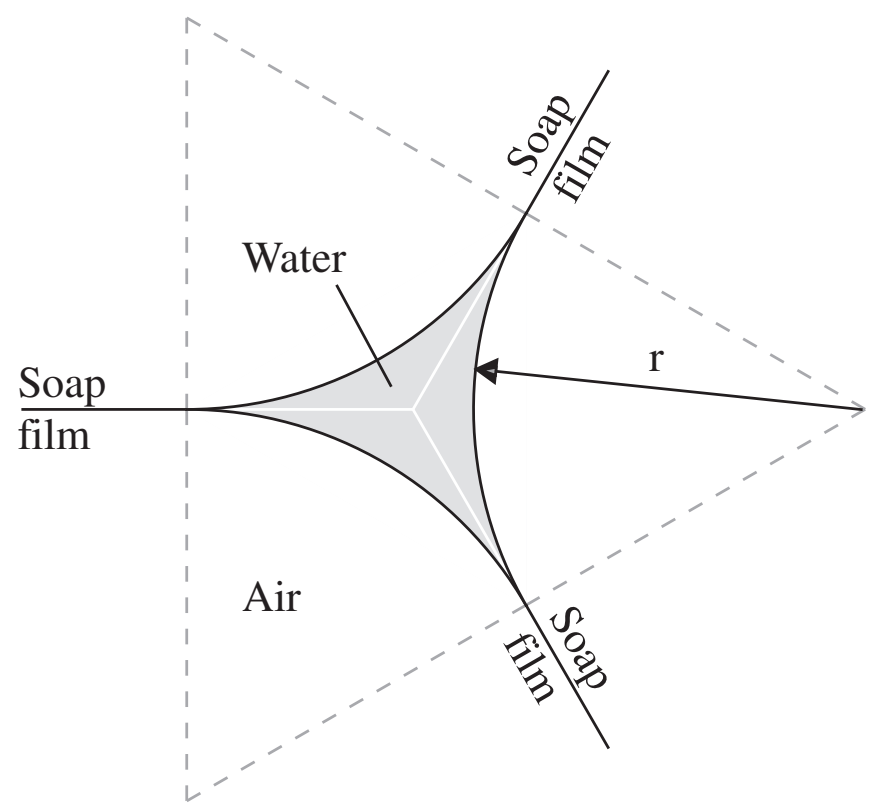

FIG. 1. Cross section of the Plateau border joining three planar soap films.

Within the triangle (dotted lines), the surface free energy, associated to the air-water interface (black lines), is less than that of the skeleton (white lines) which corresponds to the dry limit, $r \rightarrow 0$.
Idealization of liquid foams commonly consists in considering the films as surfaces of negligible thickness, the liquid being entirely contained in curved Plateau borders of triangular section at the junctions (Fig.1)[3]. This limit, convenient for a theoretical study, is that of the socalled "dry foam"; the water content approaches zero and one is left with a skeleton of interconnected thin films. In two dimensions, the Decoration Theorem states that the structure of a 2D-foam of finite liquid fraction, $\phi_{l}$, corresponds to that of the appropriate dry skeleton (i.e. a limiting structure for zero liquid fraction in which the vertices shrink to points); in other words, from the dry limit, the increase in $\phi_{l}$ leaves the overall foam geometry unchanged. The liquid accumulates in the Plateau borders and decorates the initial point-like vertices of the dry skeleton [4]. In three dimensions, there is no equivalent for the Decoration Theorem. In the following, we demonstrate theoretically that a negative line tension is associated to the accumulation of water in the Plateau border regions. By direct observation of an annular Plateau border, we give the first experimental evidence that such a line tension plays a role in the structure of simple 3Dfoams at finite liquid fraction.

\section{THEORETICAL BACKGROUND}

An increase in the amount of water in the Plateau border leads to a decrease in the total surface free energy and, as a consequence, the corresponding line tension is negative. This can be easily demonstrated by considering the case of three planar soap films, assumed to make 120-degrees angles between them and connected by a rectilinear Plateau border (Fig.1, [5]). In this case, the cross section of the air-water interface in the Plateau border region is a circle (radius $r$ ). Relative to the dry limit $(r \rightarrow 0)$, the variation of the surface free energy per unit length can be written

$$
\Delta \epsilon_{s}=\gamma(\pi-2 \sqrt{3}) r \simeq-0.32 \gamma r
$$

where $\gamma$ denotes the surface free energy of the air-water interface. The decoration energy $\Delta \epsilon_{s}$ is negative and proportional to the radius of curvature $r$. Thus, the surface free energy per unit length of Plateau border decreases when the liquid fraction increases. One can determine the corresponding line tension $T$, by considering a length $l$ of the Plateau border and by calculating the variation 
of the associated surface free energy at constant water volume, $V$. One gets the negative line tension

$$
T=\left.\frac{\partial l \Delta \epsilon_{s}}{\partial l}\right|_{V}=\frac{\Delta \epsilon_{s}}{2} .
$$

In the dry limit $(r \rightarrow 0)$, three soap films are predicted to make 120-degree angles between them at the vertices [6]. In the following, we will show both theoretically and experimentally that, because of the line tension $T$, the angles can differ significantly from 120 degrees.

\section{EXPERIMENTAL SITUATION AND RESULTS}

One can easily produce a catenoidal film by stretching soap between two horizontal annular frames. Cutting the initial catenoid in the horizontal plane with the help of a wet spatula, one can add to the system a horizontal circular soap film hanging between two catenoidal films (Fig.2). After a short transient (about 5 minutes), during which drainage occurs, the system equilibrates. Kept in contact with a humid atmosphere in order to avoid excessive drying, the equilibrated system can be observed for several minutes during which our measurements do not exhibit any systematic evolution with time. The radius $R$ of the Plateau border, which surrounds the horizontal film, is altered by changing either the diameter $D$ of the annular frames (usually $1 \mathrm{~cm}$ ) or the distance $H$ (up to $\sim 0.4 D$ ) between them [7]. The volume of water in the Plateau border, and consequently the radius $r$, depend on the preparation of the system.

Using a CCD camera (Panasonic, WV-CL700), we acquire images of the system from the side along the horizontal axis (Fig.3). These images are subsequently digitized and analyzed with the help of a PC equipped with a frame grabber board (Data Translation, DT2255) and a data analysis software (Wavemetrics, Igor Pro 4). We carefully analyzed, and then excluded, the deformations of the images induced by the optical system; we imaged a grid in the same optical conditions as the soap films and found that the only deformation is due to the small anisotropy of the CCD, which we measured to be $1.71 \pm 0.01 \%$. The measurements consist in detecting the profile $\rho(z)$ of the films, above and below the Plateau border. In order to achieve the required accuracy, the numerical procedure scans first the image from top to bottom, detecting the outline of the catenoidal films within 1 pixel; the position $\rho(z)$ is then detected within about 0.1 pixel by interpolating with a gaussian function several intensity profiles, obtained along lines normal to the initial outline. In order to exclude the possible residual tilt of the image (always less than 1 degree) and to determine accurately the axis of revolution, the profile $\rho(z)$ is detected on both the right- and left-hand sides of the image. Both the lower and upper experimental profiles are then simultaneously interpolated with two different catenoidal profiles (hyperbolic cosines), allowing the free

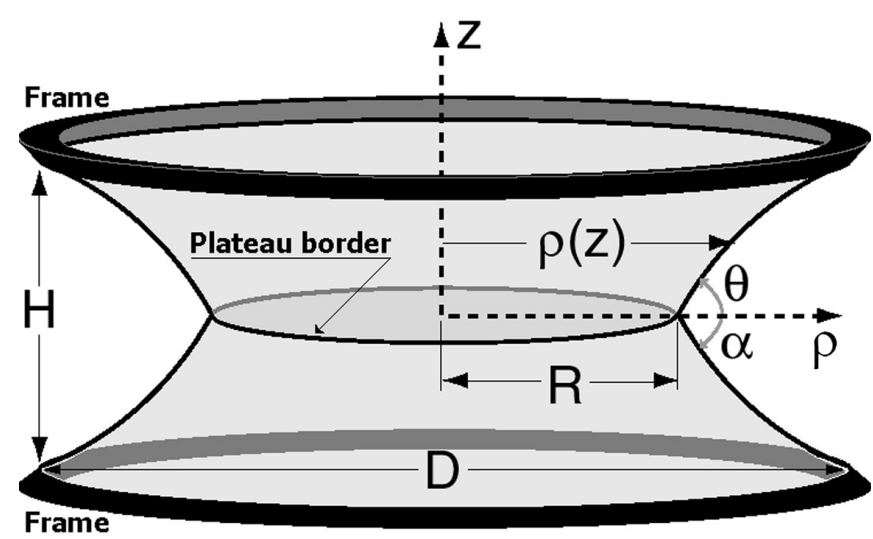

FIG. 2. Sketch of the experimental situation.

A circular soap film is suspended by two catenoidal films attached to two horizontal annular frames. The radius $R$ of the Plateau border, that connects the three films, is altered by changing either the diameter $D$ of the frames or the distance $H$ between them. The upper (resp. lower) catenoidal film makes the angle $\theta$ (resp. $\alpha$ ) with the horizontal plane. Experimentally, accurate values of the angles $\theta$ and $\alpha$ are obtained by carefully detecting the profile $\rho(z)$.

rotation of the image. We determine three quantities in the plane of the horizontal film, where the two catenoidal profiles intersect: the angle $\theta$ (resp. $\alpha$ ) that the upper (resp. lower) film makes with the horizontal plane and the radius $R$ of the Plateau border (Fig.2). We measure the radius $r$ by direct interpolation of the air-water interface observed on additional images taken at higher magnification.

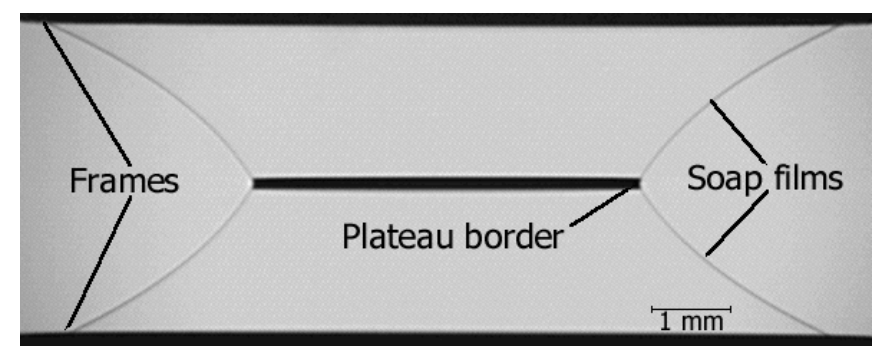

FIG. 3. Picture of the experimental situation.

Three soap films are stretched with the help of two horizontal annular frames. The central part of the structure is made of a horizontal circular film held by two catenoidal films. In this side view, the horizontal film is hidden by the thick annular Plateau border (The angle between the two catenoidal films equals $120.3 \pm 0.1$ degrees).

For large radii, $r$, the weight of water in the Plateau border is not necessarily negligible and the experimental situation needs further analysis before we can draw conclusions [Note that the upper and lower films are not symmetric with respect to the horizontal film in figure 
(3)]. Taking into account the line tension, $T$, and the weight of water per unit length, $\rho s g$, one can write the two relations imposed by the local mechanical equilibrium of the Plateau border in the horizontal plane and along the vertical

$$
\begin{aligned}
& \cos \theta+\cos \alpha=1+\frac{T}{2 \gamma R} \\
& \sin \theta+\sin \alpha=\frac{\rho s g}{2 \gamma}
\end{aligned}
$$

where $\gamma$ denotes the surface free energy of the air-water interface, $\rho$ the density of water, $g$ the acceleration due to gravity, and $s=(\sqrt{3}-\pi / 2) r^{2}$ the surface area of the Plateau-border cross section. We point out that equations (3) and (4) apply to the skeleton and must be evaluated in the plane of the horizontal film. Summing the squares of equations (3) and (4), one obtains that the angle $\beta \equiv \theta-\alpha$ satisfies

$$
\cos \beta=-\frac{1}{2}+\frac{1}{8}\left(\frac{s}{l_{c}^{2}}\right)^{2}+\frac{1}{2} \frac{T}{\gamma R}+\frac{1}{8}\left(\frac{T}{\gamma R}\right)^{2}
$$

where $l_{c}=\sqrt{\gamma / \rho g}$ is the gravitational capillary length.

According to equation (5), in the dry limit $(r \rightarrow 0)$, the angle $\beta \rightarrow 120$ deg. The weight of water tends to decrease the angle $\beta$ when the characteristic size of the Plateau border, $r$, compares to the capillary length, $l_{c}$. By contrast, the negative line tension $T$ tends to increase the angle $\beta$ when the radius of curvature $R$ approaches the typical size $r$ of the Plateau border. From equation (5), one can deduce that the correction to $\beta$ due to gravity is negligible in comparison to that due to the line tension only in the limit $l_{c} \gg \sqrt[4]{R r^{3}}$. This condition is usually not satisfied in our experimental conditions. Thus, in order to compare accurately the experimental results to the theory, one must consider the relevant quantity $\Sigma=$ $\cos (\theta)+\cos (\alpha)$ which is not altered by gravity (Eq.3). Moreover, one can better appreciate the magnitude of the line tension effects on the geometry of the structure by considering rather the angle $\beta^{*} \equiv 2 \cos ^{-1}(\Sigma / 2)$ than $\Sigma$. We expect, from equation (3) in the limit $T \ll \gamma R$,

$$
\beta^{*}=\frac{2 \pi}{3}+\left(1-\frac{\pi}{2 \sqrt{3}}\right) \frac{r}{R}+\mathcal{O}\left[\left(1-\frac{\pi}{2 \sqrt{3}}\right)^{2} \frac{r^{2}}{R^{2}}\right]
$$

Because of the small value $\left(1-\frac{\pi}{2 \sqrt{3}}\right) \sim 0.1$ of the prefactor, the non-linear terms in equation (6) are negligible in any experimental situation. The angle $\beta^{*}$ corresponds to the value of the angle $\beta \equiv \theta-\alpha$ expected from measurements in absence of gravity. In the dry limit $(r \rightarrow 0)$, $\beta^{*}=120$ degrees. Because of the negative line tension $T$, the angle $\beta^{*}$ is of about 121 degrees for $R / r=5$ and remains larger than 120.2 degrees for $R / r=20$. The agreement in figure (4) between our experimental measurements and equation (6) demonstrates clearly that the negative line tension $T$, defined by equation (2), is associated to the Plateau border region.

We point out, that the cross section of the air-water interface in the Plateau border region is not circular

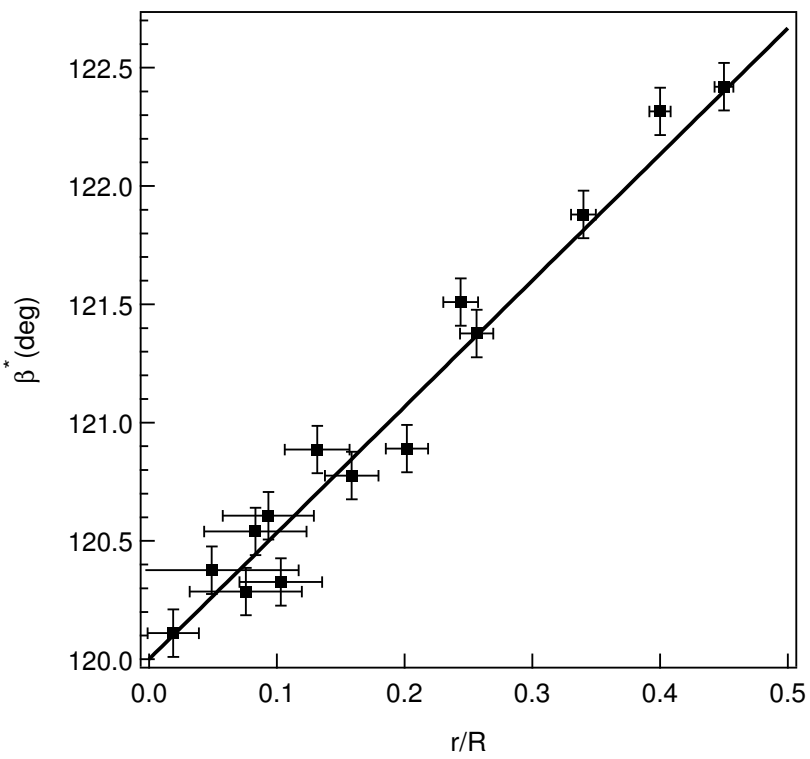

FIG. 4. Experimental angle $\beta^{*}$ vs. experimental ratio $r / R$. Circles: experimental data; Solid line: equation (6). The angles are known to within $0.1 \mathrm{deg}$ whereas the radius $r$ is measured to within 1 pixel (to be compared to $R \sim 150$ pixels).

anymore when the Plateau border is curved, because of the additional curvature $\sim 1 / R$ in the horizontal plane. Moreover, because of the line-tension $T$ itself, the angles between the films differ from 120 degrees. As a consequence, the line tension $T$ differs slightly from the value given by equation (2), which is exact only in the limit of a rectilinear Plateau border. Writing the Lagrange equation minimizing the total surface free energy of the system at constant water volume, we computed the exact shape of the air-water interface in the Plateau border region as well as that of the films in our experimental geometry (Fig.2). We found that the discrepancy between the line tension $T$ and the approximation given by equation (2) agree quantitatively to within $\sim 2.2 r / R \%$. Thus, equation (2) provides a good approximation of the line tension $T$ in most of the practical cases.

\section{CONCLUSION}

In conclusion, negative line tension is associated to finite water volumes in the Plateau borders joining soap films. This latter line tension affects the overall geometry in 3D; as observed experimentally, the angles that the films make at the Plateau border junction can differ significantly from 120 degrees. This effect, which is not due to gravity, is often neglected in the theoretical description of foams, usually studied in the dry limit, and could be important when the volume fraction of water is increased. Weaire and Kern suggested recently, that negative decoration energy and line tension must be in- 
troduced in the modelling of foams approaching the dry limit [8]. Our experimental results reinforces their theoretical approach consisting in considering a dry skeleton, whose properties are evaluated in the presence of a negative tension.

\section{ACKNOWLEDGEMENTS}

The authors would like to thank D. Weaire, N. Kern, F. Graner and V. Bergeron for stimulating discussions on foam physics. One of us (A. Z.) would like to thank the Centre National de la Recherche Scientifique (France) and the Ministère des Affaires Etrangères (France, Polonium $05844 \mathrm{XG}$ ) for financial support.
[1] Weaire D. and Hutzler S., "The physics of foams", Clarendon Press, Oxford (1999).

[2] Duran J., Sands, Powders, and Grains : An Introduction to the Physics of Granular Materials (Springer, New York, 2000); Jaeger H. M., Nagel S. R., and Behringer R. P., Rev. Mod. Phys. 681259 (1996); Kadanoff L. P., Rev. Mod. Phys. 71435 (1999); Herrmann H. J., Luding S., and Cafiero R., Physica A 29593 (2001).

[3] Plateau J., "Statique expérimentale et théorique des liquides soumis aux seules forces moléculaires", GauthierVillars, Paris (1873).

[4] Weaire D., Phil. Mag. Lett. 79, 491 (1999).

[5] Krotov V. V. and Rusanov A. I., Mendeleev Commun. 5, 177 (1998).

[6] Almgren F. J. and Taylor J., Sci. Amer. 235, 82 (1976).
[7] For larger distance $H$ between the frames, the system is unstable and the collapse of the structure leads to the formation of one planar film on each of the frames and of a droplet. This collapse, similar to that of a simple catenoid [10], as well as the response of the structure to a mechanical perturbation will be subjects of further publications.

[8] Kern N. and Weaire D., Phil. Mag. 83, 2973 (2003).

[9] Hildebrandt S. and Tromba A., "Mathematics and optimal form", Scientific American Library, New-York (1985).

[10] Chen Y.-J. and Steen P. H., J. Fluid Mech. 341, 245 (1997); Cryer S. A. and Steen P. H., J. Coll. Int. Sci. 154, 276 (1992). 